\title{
Classification of Maojian Teas from Different Geographical Origins by Micellar Electrokinetic Chromatography and Pattern Recognition Techniques
}

\author{
Nengsheng Ye, ${ }^{\dagger}$ Liqin Zhang, and Xuexin Gu \\ Department of Chemistry, Capital Normal University, Beijing 100048, P. R. China
}

\begin{abstract}
A micellar electrokinetic chromatography (MEKC) method was applied for the identification of geographical origins of Chinese green teas. Under the optimized conditions, chromatographic profiling of collected Maojian tea samples was obtained. Based on MEKC-UV profiling, twenty-four tea samples were successfully differentiated according to the relative peak areas of selected peaks in the chromatograms. Tea samples from Hubei and Henan provinces were classified correctly by hierarchical cluster analysis model (HCA) and principal component analysis (PCA). The application of linear discriminant analysis (LDA) gave correct assignation percentages of $100 \%$ for the training set and the prediction set. The overall results demonstrated that MEKC with pattern recognition could be successfully applied to discriminate Maojian teas according to their geographical origins.
\end{abstract}

(Received November 25, 2010; Accepted May 11, 2011; Published July 10, 2011)

\section{Introduction}

Green tea is the most popular and widely consumed beverage in the world. It is well known that green tea has important physiological properties and potential health benefits due to the presence of chemical compounds, such as polyphenols, amino acids, and vitamins. ${ }^{1}$ Maojian tea is an important variety of Chinese green teas, and is produced in different areas. The quality of Maojian teas is different because of the climate and growing surroundings of tea plantations. Some famous Maojian teas are popular in China because of their high quality, such as Xinyang Maojian in Henan province and Wufengshan Maojian in Hubei province. The production of the particular plantations in these areas means these producers can ask a higher price for their specific product than the average price. This leads to a temptation for unscrupulous producers to fraudulently label their product as coming from one of these areas to take advantage of this higher price. However, the professional tea tasters have expert knowledge on tea, and they are able to identity and describe the various attributes of tea liquor, the origins between different teas. But the opinions from tea tasters may be different according to personal preferences. There is a need to protect both buyer and seller by developing analytical methods that can be used to identify the provenance of teas.

The discrimination of tea products from different geographical origins is restricted to the employment of chemical methods. ${ }^{2,3}$ The content of the mineral elements in teas was determined for the identification of their geographical origins. ${ }^{4-6}$ Some spectrometric methods were used for the geographical analysis of teas, such as flourier transformation near-infrared (FT-NIR). ${ }^{7}$ Electronic tongue and electronic nose were applied for the

† To whom correspondence should be addressed.

E-mail: yensh@mail.cnu.edu.cn classification and quality control of tea samples. ${ }^{8}$ Though the chemical compositions of various green tea samples from different origins are similar, it is possible to differentiate them by some parameters (chemical components) that can be obtained by chromatographic methods, such as capillary electrophoresis (CE),${ }^{9}$ gas chromatography (GC),${ }^{10,11}$ and high performance liquid chromatography (HPLC). ${ }^{12-14}$ Our previous paper reported the application of micellar electrokinetic chromatography (MEKC) with a LIF detector for the geographical classification of teas from different areas in China. ${ }^{15}$ Some important chemical compositions (such as catechins) were not detected by LIF detector without derivatization. But these compositions will play an important role in the classification of tea samples. MEKC-UV method was used for the determination of catechins in green tea samples. ${ }^{16}$ In this work a MEKC-UV approach was used for chromatographic profiling of green tea samples from different geographical origins, and then linear discriminant analysis (LDA) was adopted to develop a discriminant model of tea samples with categories. Meanwhile, unsupervised pattern recognition algorithms, included hierarchical cluster analysis (HCA) and principal component analysis (PCA), were also used for the discrimination of tea samples used in this work.

\section{Experimental}

\section{Reagents and materials}

Sodium dodecyl sulfate (SDS) and methanol (LC grade) were obtained from Sigma-Aldrich. Other reagents were analytical grade. All solutions were prepared with Milli-Q water.

Maojian tea samples were obtained from local tea stores and stored in a cool and dry place. All collected tea samples were harvested in the spring of 2009. Among these tea samples, thirteen teas were collected from Hubei province, and 11 other tea samples were from Henan province. 
Table 1 Analytical method validation results of inter-day and intra-day precision

\begin{tabular}{rccccc}
\hline & \multicolumn{2}{c}{$\begin{array}{c}\text { RSD of relative } \\
\text { migration time, } \%\end{array}$} & & \multicolumn{2}{c}{$\begin{array}{c}\text { RSD of relative } \\
\text { peak area, } \%\end{array}$} \\
\cline { 2 - 3 } \cline { 5 - 6 } No. & $\begin{array}{c}\text { Inter-day } \\
(n=9)\end{array}$ & $\begin{array}{c}\text { Intra-day } \\
(n=6)\end{array}$ & & $\begin{array}{c}\text { Inter-day } \\
(n=9)\end{array}$ & $\begin{array}{c}\text { Intra-day } \\
(n=6)\end{array}$ \\
\hline 1 & 0.70 & 0.87 & & 5.23 & 3.43 \\
2 & 0.86 & 0.78 & & 5.01 & 4.87 \\
3 & 0.91 & 0.90 & & 5.12 & 4.67 \\
4 & 0.79 & 0.86 & & 4.87 & 4.90 \\
5 & 0.43 & 0.64 & & 1.34 & 2.12 \\
6 & 1.04 & 0.66 & & 5.04 & 3.54 \\
7 & 0.77 & 0.81 & & 4.91 & 4.90 \\
8 & 0.64 & 0.54 & & 2.43 & 1.52 \\
9 & 0.78 & 0.69 & & 3.76 & 3.09 \\
10 & 0.82 & 0.92 & & 4.37 & 4.75 \\
11 & 0.94 & 0.86 & & 3.92 & 1.83 \\
12 & 1.11 & 0.88 & 5.17 & 2.31 \\
13 & 0.93 & 0.67 & 2.39 & 2.21 \\
14 & 0.67 & 0.34 & 4.65 & 3.45 \\
15 & 1.06 & 0.61 & 4.88 & 2.18 \\
\hline
\end{tabular}

Table 2 Analytical method validation results of reproducibility $(n=5)$

\begin{tabular}{ccc}
\hline Peak No. & $\begin{array}{c}\text { RSD of relative } \\
\text { migration time, } \%\end{array}$ & $\begin{array}{c}\text { RSD of relative } \\
\text { peak area, } \%\end{array}$ \\
\hline 1 & 0.68 & 3.15 \\
3 & 0.55 & 5.08 \\
4 & 0.90 & 3.34 \\
5 & 0.42 & 2.54 \\
6 & 0.21 & 3.94 \\
7 & 1.21 & 4.23 \\
8 & 0.35 & 1.44 \\
9 & 0.81 & 2.44 \\
10 & 0.43 & 4.39 \\
11 & 0.35 & 4.39 \\
12 & 0.57 & 4.34 \\
13 & 0.53 & 1.59 \\
14 & 0.34 & 8.34 \\
15 & 0.65 & 4.04 \\
\hline
\end{tabular}

\section{Apparatus}

A P/ACE MDQ capillary electrophoresis system (Beckman Coulter, USA) equipped with a photodiode array detector was used to perform the separation. An uncoated fused silica capillary (Yongnian, China) with a total length of $60 \mathrm{~cm}(50 \mathrm{~cm}$ to the detector window, $75 \mu \mathrm{m}$ i.d.) was used as the separation column. 32 Karat software (Edition 8.0, Beckman Coulter, USA) was used for the operation of CE system and data processing. All classification was implemented with Matlab V7.0 software (Mathworks, USA).

\section{MEKC conditions}

A $20 \mathrm{mM}$ phosphate solution ( $\mathrm{pH} \mathrm{6.3)} \mathrm{containing} 25 \mathrm{mM}$ SDS was used as background electrolyte (BGE). A voltage of $12 \mathrm{kV}$ was applied and the detection was performed at $210 \mathrm{~nm}$. The capillary temperature was set at $25^{\circ} \mathrm{C}$. The samples were injected by 0.5 psi pressure for $5 \mathrm{~s}$.

Each new capillary was initialized with methanol for $5 \mathrm{~min}$, $0.1 \mathrm{M} \mathrm{NaOH}$ for $30 \mathrm{~min}$, and deionized water for $10 \mathrm{~min}$, in order. The rinse pressure was set at 15 psi. In between the runs,

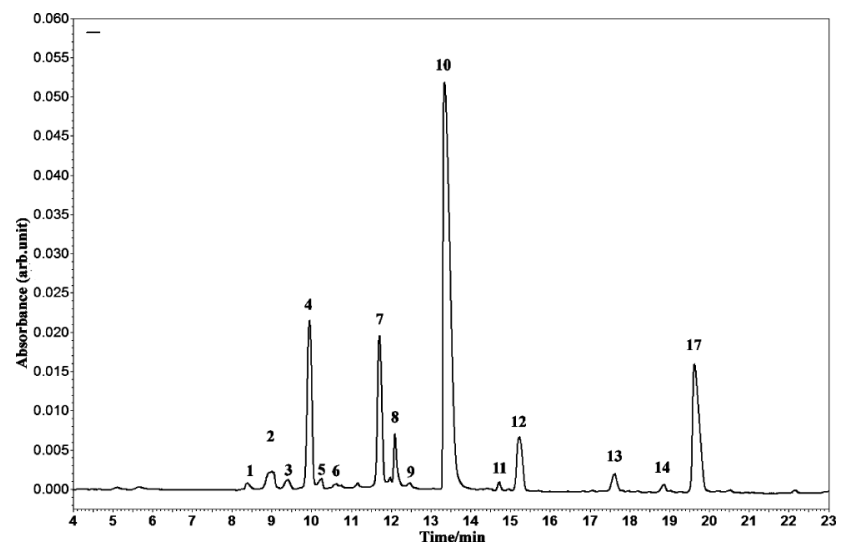

Fig. 1 Representative chromatogram of MEKC-UV profiling of Maojian tea (sample No. HN-1). MEKC-UV conditions: uncoated fused silica capillary $(50 \mathrm{~cm} / 60 \mathrm{~cm}, 75 \mu \mathrm{m})$; BGE, $25 \mathrm{mM}$ SDS in a $20 \mathrm{mM}$ phosphate solution ( $\mathrm{pH} 6.3$ ); separation voltage, $12 \mathrm{kV}$; UV detection, $210 \mathrm{~nm}$; temperature, $25^{\circ} \mathrm{C}$; injection, 0.5 psi for $5 \mathrm{~s}$. Peak identification: 7, EGC; 10, EGCG; 11, EC; 12, GCG; 15, ECG.

each capillary was rinsed with $0.1 \mathrm{M} \mathrm{NaOH}$ and deionized water for $1 \mathrm{~min}$, and followed with the running buffer for $8 \mathrm{~min}$. To ensure reproducibility, we renewed the buffer after two electrophoretic runs. All solutions were filtered through a $0.45-\mu \mathrm{m}$ pore size filter.

\section{Sample preparation}

Taking the heterogeneity of tea samples into account, we first ground each Maojian tea samples. Tea leaves were put into a mortar and ground until they turned into a fine powder. Then, $0.5000 \pm 0.0005 \mathrm{~g}$ of tea powder were weighed and placed into a $250-\mathrm{mL}$ beaker for extraction. A $75-\mathrm{g}$ volume of deionized water at $80^{\circ} \mathrm{C}$ was added, and then mixture was stirred for $10 \mathrm{~min}$. The tea brew was filted through a $0.45-\mu \mathrm{m}$ filter, and tea infusions were sealed tightly and stored at $2-4^{\circ} \mathrm{C}$.

\section{Results and Discussion}

\section{Optimization of MEKC conditions}

In order to achieve a rapid separation, we optimized the BGE according to the previous reports. ${ }^{17,18}$ SDS-phosphate solution was chosen as BGE, and then the effects of the concentration of phosphate solution, the $\mathrm{pH}$ of $\mathrm{BGE}$ and the concentrations of SDS in BGE on the chromatographical fingerprint were investigated. Catechins were unstable and completely degraded within a few minutes in alkaline media $(\mathrm{pH}>8.0)$. The stability of catechins was increased in acidic solutions, but the migration times of analytes were increased because of the lower electroosmotic flow. When the $\mathrm{pH}$ was increased from 5.8 to 7.0 , the migration time was decreased, but the response signal value also decreased. The $\mathrm{pH}$ of BGE was chosen to be $\mathrm{pH}$ 6.3.

After the concentration of phosphate was adjusted, the viscosity of the solution was increased with the increasing of the concentration of phosphate, resulting in the decreased velocity of electroosmotic flow and longer separation time, and the concentration of phosphate was selected to be $20 \mathrm{mM}$. For rapid separation, better peak resolutions and fewer joule-heating problems, SDS concentration was selected to be $25 \mathrm{mM}$. After systemic optimization, BGE was as follows: a $25 \mathrm{mM}$ SDS in $20 \mathrm{mM}$ phosphate $(\mathrm{pH} 6.3)$ solution. 


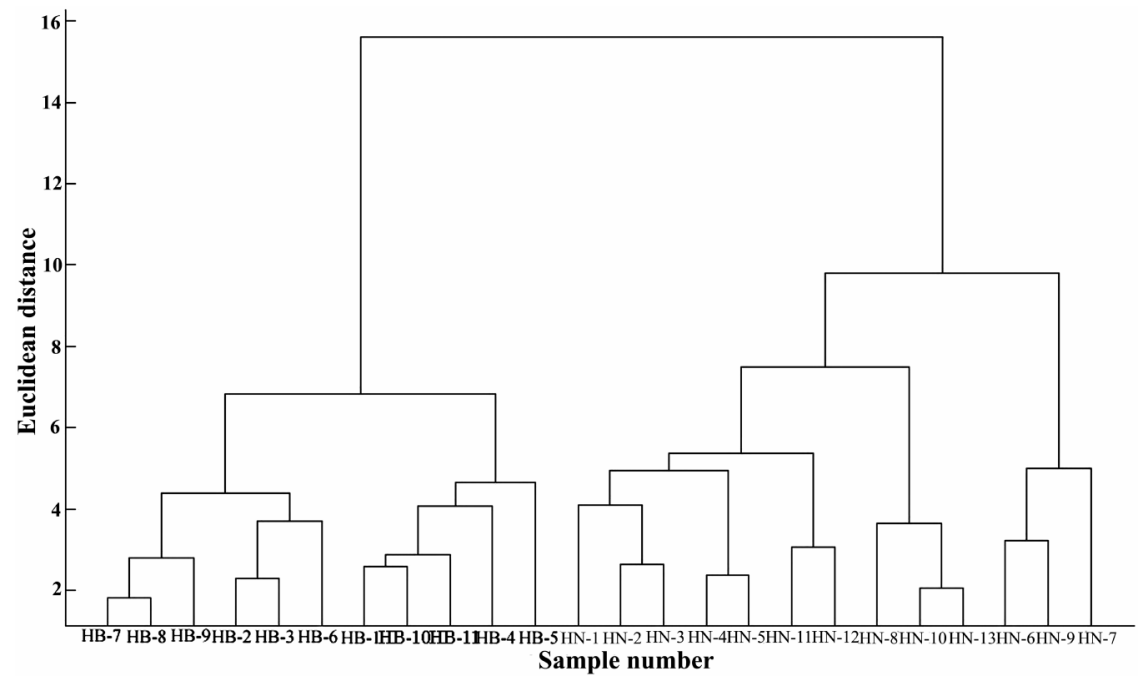

Fig. 2 Clustering of Maojian tea samples according to the relative peak areas of fifteen selected peaks in chromatograms.

\section{Methodological validation}

A Maojian tea sample from Henan province (No. HN-1) was used for the precision test, the inter-day precision of the developed method was evaluated by evaluating one sample that was injected three times every day for three days; and the intra-day precision was evaluated for six injections of the same tea infusion. The RSDs of the inter-day precision $(n=9)$ and intra-day $(n=6)$ precision for fifteen selected peaks are listed in Table 1.

The reproducibility precision was evaluated by running five replicate samples on the same day. The RSDs of reproducibility of fifteen selected peaks are listed in Table 2.

\section{Chromatographic fingerprint of Maojian teas}

Under the optimal MEKC-UV conditions, chromatograms of the collected Maojian samples with same type from different origins were obtained. The chromatograms were characteristic, and the same variety of Maojian teas from different origins showed similar peak shape. A typical chromatogram of Maojian tea is shown in Fig. 1. Compared with the migration times of Maojian tea sample and standard substances, five peaks numbered with 7, 10, 11, 12, and 15 in Fig. 1 was identified as epigallocatechin (EGC), epigallocatechin gallate (EGCG), epicatechin (EC), gallocatechin gallate (GCG) and epicatechin gallate (ECG), respectively. In the MEKC chromatogram the other 10 peaks were not identified, because of the lack of their standard substances, but the characterization of MEKC profiling can be described by their relative peak areas. Selected peak (No. 4) in chromatograms was used as a reference peak because of its representative peak shape and strong peak intensity. The relative peak areas $\left(S_{\mathrm{r}}\right)$ of the other 14 selected peaks were obtained and are listed in Table S1 (Supporting Information), and these data were used for the differentiation of collected tea samples from different origins by different pattern recognition techniques.

\section{HCA model}

HCA is used to assign a set of observations into subsets (called clusters) so that observations in the same cluster are similar in some senses. In this study the cluster trees were achieved according to the distances matrices by using the ward

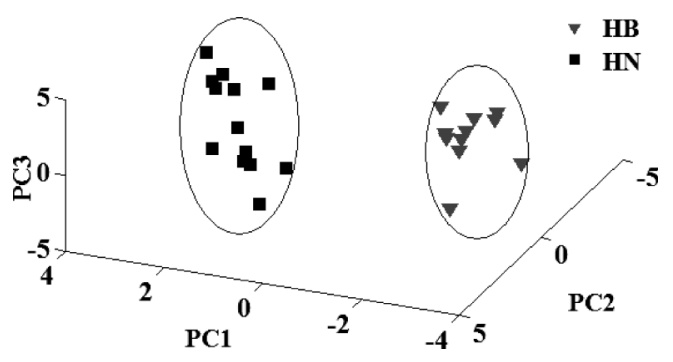

Fig. 3 Scores of the Maojian tea samples in the three dimensions formed by PCA.

algorithm. As shown in Fig. 2, a clear discrimination between samples from Henan and Hubei provinces was obtained by HCA. Twenty-four samples of Maojian teas from different places in Henan and Hubei provinces were classified into two groups according to their geographical origins.

\section{PCA model}

PCA was used to achieve a reduction of dimension and to observe a primary evaluation of the between-class similarity. To visualize the cluster trends of tested Maojian tea samples, a score plot was obtained by using some top principle components from 15 variables, and keeping most of the original information content in the data set. As shown in Fig. 3, the five principal components represented about $89 \%$ of the total variability, where PC1, PC2, PC3, PC4 and PC5 contributed 40.0, 26.1, $10.3,7.2$ and $5.8 \%$ of the total variance, respectively. On the 3D plot, Maojian tea samples from Henan province (HN) formed a group that included thirteen samples; these samples were marked by squares. The other Maojian tea samples from Hubei province (HB) group included eleven samples; these samples were marked by inverted triangles. Maojian teas showed considerable differences in their own chemical characteristics according to their geographical origins. The differences between chemical characteristics of Maojian teas were reasonably differentiated according to MEKC profiling. Therefore, the MEKC data showed the cluster trend of Maojian 
Table 3 Number and geographical origins of Maojian tea samples in the training and prediction sets

\begin{tabular}{ccc}
\hline \multirow{2}{*}{$\begin{array}{c}\text { Geographical } \\
\text { origin }\end{array}$} & \multicolumn{2}{c}{ Numbers of sample } \\
\cline { 2 - 3 } & Training set & Prediction set \\
\hline Henan & 10 & 3 \\
Hubei & 9 & 2 \\
\hline
\end{tabular}

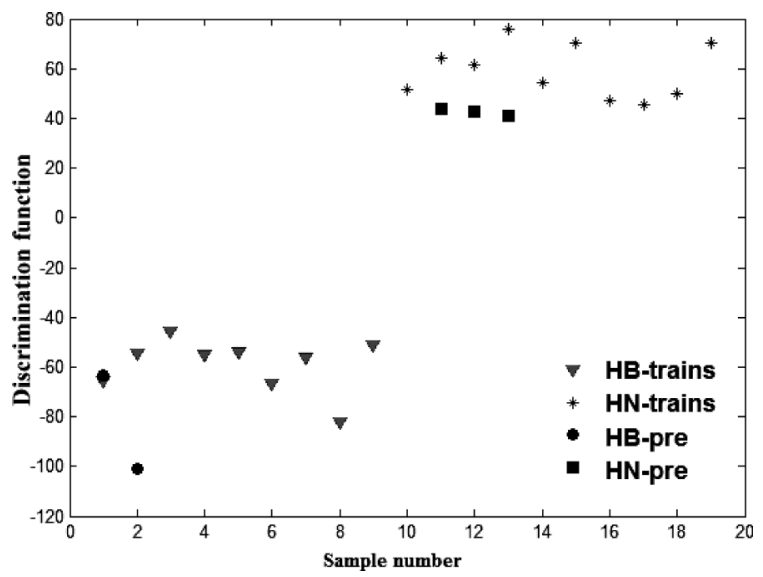

Fig. 4 Scatter plot of Maojian tea samples in the discriminant functions by LDA.

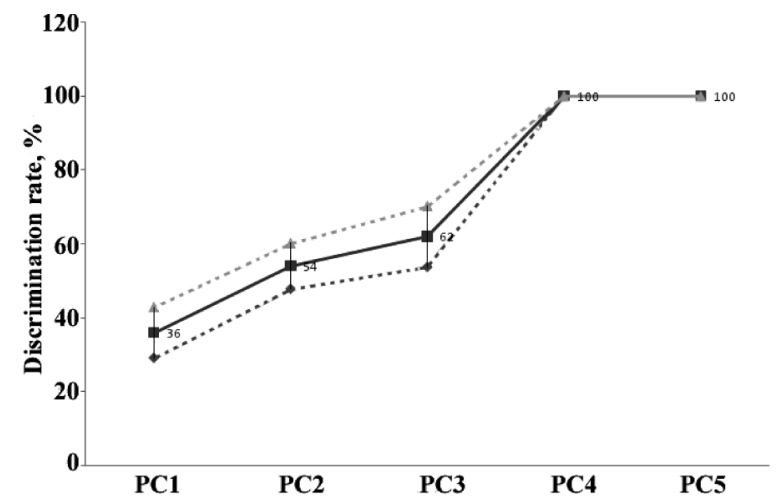

Fig. 5 Discrimination rates of LDA model according to different PCs.

tea samples according to geographical origins by means of PCA.

\section{Discrimination model of supervised LDA}

The goal of this work is to discriminate Maojian teas according to their geographical origins by MEKC profiling. All Maojian tea samples were divided into a training set $(79.2 \%, 19$ samples) and a prediction set $(20.8 \%, 5$ samples) by Kennard-Stone (KS) algorithm. In order to keep the reliability of data, the sample was randomly extracted $(n=20)$ by KS method. The distributions of all Maojian tea samples in the training and prediction sets were listed in Table 3 .

Supervised LDA model was adopted to classify all Maojian tea samples into groups of known geographical origins. It was applied to develop a set of discriminating functions; derived from values of 15 variables (relative peak area, $S_{\mathrm{r}}$ ) according to the maximization of the F-ratio between points belonging to the categories. The discrimination function (DF) was calculated as follows;

$$
\begin{aligned}
\mathrm{DF}= & 101.708 S_{1}-460.0011 S_{2}-654.4447 S_{3}+36.5756 S_{4}+ \\
& 14.9071 S_{5}+142.805 S_{6}-40.3463 S_{7}-112.8031 S_{8}- \\
& 17.8906 S_{9}+3.557 S_{10}+36.63 S_{11}+142.68 S_{12}+ \\
& 0.1349 S_{13}-44.20 S_{14}-436.51 S_{15} .
\end{aligned}
$$

Figure 4 shows a plot of samples obtained when using DF as axes. All Maojian tea samples were correctly classified, tea samples from Henan province (marked with HN in Fig. 4) were located at the upper right, and tea samples from Hubei province (marked with HB) were found at the lower left. The number of principal component factors was important for the performance of LDA, and the LDA discrimination rates by cross-validation were used to optimize the number of PC4. The discrimination rates of LDA model according to different PCs are shown in Fig. 5. The optimal number of PCs was determined according to the highest discrimination rates by cross-validation. When PC4 was used for LDA, the discrimination rate was $100 \%$ in both the training set and the prediction set.

\section{Comparison of different models}

To highlight the good performance in discrimination of Maojian teas according to their geographical origin, we have applied pattern recognition methods involving display methods such as PCA and HCA, and we have used supervised learning procedure LDA in this work.

The classifications of tea samples from Henan and Hubei provinces were $100 \%$ accurate in total by PCA, HCA and LDA. However, both HCA and PCA are both non-supervised models, and they only give the cluster trend of Maojian tea samples according to geographical origins. LDA, as a supervised technique, is a useful complement to PCA, and was applied to evaluate differences in tea samples from different geographical origins.

\section{Conclusions}

This work, although limited by the number of samples available, clearly shows that the differentiation and classification of Maojian tea samples from Henan and Hubei provinces in China is possible using the MEKC profiling and applying pattern recognition techniques. Three pattern recognition methods (LDA, HCA and PCA) can be used to develop the discrimination models for Chinese Maojian teas in this work.

\section{Acknowledgements}

The work was supported by the National Natural Science Foundation of China (21005050), the Scientific Research Common Program of Beijing Municipal Commission of Education (KM201010028009) and the Funding Project for Academic Human Resources Development in Institutions of Higher Learning under the Jurisdiction of Beijing Municipality (PHR20100718 and PHR201108147).

\section{Supporting Information}

The relative peak areas $\left(S_{\mathrm{r}}\right)$ are listed in Table S1. This material is available free of charge on the Web at http://www.jsac.or.jp/ analsci/. 


\section{References}

1. M. S. Butt and M. T. Sultan, Crit. Rev. Food Sci., 2009, 49, 463.

2. A. Alcazar, O. Ballesteros, J. M. Jurado, F. Pablos, M. J. Martin, J. L. Vilches, and A. Navalon, J. Agric. Food Chem., 2007, 55, 5960 .

3. J. H. Jang, E. S. Kim, S. Y. Wu, J. L. Lu, H. L. Liang, Y. Y. Du, C. Lin, and Y. R. Liang, Food Sci. Biotechnol., 2008, 17, 1016.

4. Y. X. Chen, M. G. Yu, J. Xu, X. C. Chen, and J. Y. Shi, J. Sci. Food Agric., 2009, 89, 2350.

5. P. L. Fernandez-Caceres, M. J. Martin, F. Pablos, and A. G. Gonzalez, J. Agric. Food Chem., 2001, 49, 4775.

6. T. S. Pilgrim, R. J. Watling, and K. Grice, Food Chem., 2010, $118,921$.

7. Q. S. Chen, J. W. Zhao, and H. Lin, Spectrochim. Acta, Part A, 2009, 72, 845 .

8. W. He, X. S. Hu, L. Zhao, X. J. Liao, Y. Zhang, M. W.
Zhang, and J. H. Wu, Food Res. Int., 2009, 42, 1462.

9. R. Gotti, S. Furlanetto, S. Lanteri, S. Olmo, A. Ragaini, and V. Cavrini, Electrophoresis, 2009, 30, 2922.

10. L. F. Wang, J. Y. Lee, J. O. Chung, J. H. Baik, S. So, and S. K. Park, Food Chem., 2008, 109, 196.

11. W. Pongsuwan, T. Bamba, T. Yonetani, A. Kobayashi, and E. Fukusaki, J. Agric. Food Chem., 2008, 56, 744.

12. P. L. Fernandez, A. Lopez, F. Pablos, A. G. Gonzalez, and M. J. Martin, Microchim. Acta, 2003, 142, 79.

13. P. L. Fernandez, M. J. Martin, A. G. Gonzalez, and F. Pablos, Analyst, 2000, 125, 421.

14. Y. He, X. K. Hong, and Z. H. Wang, Chin. J. Anal. Chem., 2006, 34, 843 .

15. N. S. Ye, Chromatographia, 2010, 71, 529.

16. G. Q. Song, J. M. Lin, F. Qu, and W. J. Dong, Chin. J. Chem., 2003, 21, 1325.

17. C. N. Chen, C. M. Liang, J. R. Lai, Y. J. Tsai, J. S. Tsay, and J. K. Lin, J. Agric. Food Chem., 2003, 51, 7495.

18. D. J. Weis, E. J. Austria, C. R. Anderton, R. Hompesch, and A. Jander, J. Chromatogr., A, 2006, 1117, 103. 